\title{
Nucleotide sequence of the $\alpha$-amylase-pullulanase gene from Clostridium thermohydrosulfuricum
}

\author{
Hannes Melasniemi, ${ }^{*}$ Marja Paloheimo and leena Hemiö \\ Research Laboratories, Alko Ltd, POB 350, SF-00101, Helsinki, Finland \\ (Received 8 September 1989; revised 9 November 1989; accepted 21 November 1989)
}

\begin{abstract}
The nucleotide sequence of the gene (apu) encoding the thermostable $\alpha$-amylase-pullulanase of Clostridium thermohydrosulfuricum was determined. An open reading frame of $4425 \mathrm{bp}$ was present. The deduced polypeptide $\left(M_{r}\right.$ 165600), including a 31 amino acid putative signal sequence, comprised 1475 amino acids, with no cysteine residues. The structural gene was preceded by the consensus promoter sequence TTGACA TATAAT, a putative regulatory sequence and a putative ribosome-binding sequence AAAGGGGG. The codon usage resembled that of Bacillus genes. The deduced sequence of the mature apu product showed similarities to various amylolytic enzymes, especially the neopullulanase of Bacillus stearothermophilus, whereas the signal sequence showed similarity to those of the $\alpha$-amylases of $B$. stearothermophilus and $B$. subtilis. Three regions thought to be highly conserved in the primary structure of $\alpha$-amylases could also be distinguished in the apu product, two being partly 'duplicated' in this $\alpha-1,4 / \alpha-1,6$-active enzyme.
\end{abstract}

\section{Introduction}

$\alpha$-Amylases (EC 3.2.1.1) and pullulanases (EC 3.2.1.41) (Fogarty, 1983) are used industrially in the processing of starch. $\alpha$-Amylases degrade starch by cleaving its internal $\alpha-1,4$ linkages and usually produce as final products a mixture consisting of maltose, maltooligosaccharides and $\alpha$-limit dextrins, the last containing all the $\alpha-1,6$ branch sites initially present in the starch. Pullulanases debranch starch by cleaving the $\alpha-1,6$ linkages in its amylopectin component. When used in combination with a pullulanase, $\alpha$-amylases leave virtually no limit dextrin, and higher yields of the desired end products can be obtained.

Bacillus subtilis (Takasaki, 1987), Thermoanaerobium brockii (Coleman et al., 1987), Thermoanaerobium sp. (Plant et al., 1987) Clostridium thermohydrosulfuricum (Melasniemi, 1988) and Bacillus circulans (Sata et al., 1989), produce amylolytic enzymes having both $\alpha-1,4$ and $\alpha-1,6$ activities in the same molecule. The gene for

\footnotetext{
Abbreviations: apu, $\alpha$-amylase-pullulanase gene of $C$. thermohydrosulfuricum; DSM, Deutsche Sammlung von Mikroorganismen und Zellkulturen.

The nucleotide sequence data reported in this paper have been submitted to GenBank and have been assigned the accession number M28471.
}

the $\alpha$-amylase-pullulanase of $C$. thermohydrosulfuricum was cloned in Escherichia coli (Melasniemi \& Paloheimo, 1989) as an initial step towards producing the enzyme in an industrially better-suited host organism. Both to test different promoter and signal sequence combinations and to understand better the $\alpha$-amylase/pullulanase character of the enzyme and the relation between the enzyme and its satellite protein (Melasniemi, 1988; Melasniemi \& Paloheimo, 1989), the gene (apu) coding for the $\alpha$-amylase-pullulanase was sequenced as reported in this paper.

\section{Methods}

Plasmids and phages. The plasmids pALK351 and pALK353 containing the $\alpha$-amylase-pullulanase (apu) gene on a $7 \mathrm{~kb}$ EcoRI insert (Melasniemi \& Paloheimo, 1989) were used as sources of DNA in the sequence determination. For the preparation of the plasmid DNA, the method of Birnboim \& Doly (1979) was used. The phage vectors used for sequencing were M13mp18 and M13mp19 (Norrander et al., 1983).

Bacterial strains and culture conditions. E. coli JM109 (YanischPerron et al., 1985) harbouring pALK351 or pALK353 (DSM 5355) were grown in Luria-Bertani medium (Maniatis et al., 1982) containing ampicillin $\left(50 \mu \mathrm{g} \mathrm{ml}^{-1}\right)$. E. coli JM109 used for the transfection of M13 phage was maintained on M9 plates (Maniatis et al., 1982) containing thiamin $\left(15 \mu \mathrm{g} \mathrm{ml}^{-1}\right)$ and grown in Luria-Bertani medium.

DNA sequencing. Single-stranded DNA for sequencing was obtained by the M13 procedure of Messing (1983). The apu gene was cloned in M13mp18 and M13mp19 as several restriction fragments using restriction endonucleases EcoRI, BamHI, PstI, HindIII, AccI, KpnI 
and $S p h \mathrm{I}$ and single-stranded DNA was purified. The DNA was sequenced by the dideoxy chain-termination method of Sanger $e t$ al. (1977) using a Sequenase kit (United States Biochemical Corporation) and $\left[\alpha^{-35}\right.$ S $]$ thio-dATP (Amersham). The universal 17-mer primer included in the kit and 17-mer oligonucleotides synthesized by a $381 \mathrm{~A}$ DNA Synthesizer (Applied Biosystems) were used as primers. The whole sequence was determined in both directions with overlapping partial sequences on both strands. For the analysis of the DNA sequence the MicroGenie computer program (Beckman) was used.

\section{Results and Discussion}

\section{Nucleotide sequence and deduced amino acid sequence}

In previous experiments the coding and regulatory sequences of the $C$. thermohydrosulfuricum apu gene were localized to a $6.2 \mathrm{~kb}$ BamHI-EcoRI DNA fragment (Melasniemi \& Paloheimo, 1989). The determined nucleotide sequence of the gene, together with the deduced amino acid sequence, is shown in Fig. 1. Only one long open reading frame was present. It codes for a polypeptide with 1475 amino acids and a calculated $M_{\mathrm{r}}$ of 165600 . This is close to the $M_{\mathrm{r}}(165000)$ determined by SDS-PAGE for the biggest $a p u$ polypeptide produced in E. coli (Melasniemi \& Paloheimo, 1989) and compatible with the $M_{\mathrm{r}}(180000-190000)$ observed for the extracellular $\alpha$-amylase-pullulanase produced by $C$. thermohydrosulfuricum (Melasniemi, 1988), considering its carbohydrate content of $11-12 \%$.

The N-terminal end of the deduced apu gene product (Fig. 1) comprised a 31 amino acid sequence with the characteristics of a typical signal sequence (McConnell et al., 1986): three positively charged amino acids near the $\mathrm{N}$-terminus, a hydrophobic core, the consensus signal peptidase cleavage sequence Ala-X-Ala and in addition a helix-breaking proline preceding the cleavage site. The signal sequence of the deduced apu product (Fig. 1) was followed by the $\mathrm{N}$-terminal amino acid sequence of the extracellular enzyme purified from $C$. thermohydrosulfuricum (Melasniemi, 1988). The $\mathrm{N}$-terminal amino acid sequence earlier reported contained an Ile residue at position +2 , but the nucleotide sequence clearly indicates a $\mathrm{Thr}$ residue at this position. However, the ATA codon for Ile can be changed to the ACA codon for Thr by a single point mutation. Because of this the DNA sequence at this point must be checked from another clone.

The structural gene was preceded by a sequence identical to the $E$. coli and $B$. subtilis $\sigma^{43}$ consensus promoter sequences (Rosenberg \& Court, 1979; McConnell et al., 1986), the sequence TTGACA being followed by two TATAAT sequences after 10 and 17 nucleotides, respectively (Fig. 1). This putative promoter sequence was preceded by a 13 nucleotide inverted repeat (Fig. 1) having the characteristics of a transcription terminator (Rosenberg \& Court, 1979). This inverted repeat was the longest found in the whole sequence. Some 40 nucleotides downstream from the putative promoter started a seven nucleotide inverted repeat (AAATGTGTTTTTATCACATTT; Fig. 1) resembling the consensus sequence (AANTGTGANNTNNNNCANATTN) for binding of the CAMP-catabolite activator protein complex of $E$. coli (Crombrugghe et al., 1984). The resemblance of the apu inverted repeat to a known bacterial regulatory protein binding site makes it a candidate for the site mediating the observed catabolite repression (Hyun \& Zeikus, 1985; Melasniemi, 1987) of the apu gene. At a distance of seven nucleotides in front of the gene was the sequence AAAGGGGG (Fig. 1), exhibiting strong complementarity to the $16 \mathrm{~S}$ rRNA of Bacillus stearothermophilus and B. subtilis (McConnell et $a l ., 1986)$. The TAG stop codon terminating the $a p u$ open reading frame is followed by three other TAG codons in the same reading frame, one, 10 and 15 codons later (Fig. 1 ), ensuring termination of translation of the gene.

The $\alpha$-amylase-pullulanase satellite protein is a small protein occurring in a firm, apparently equimolar association with the extracellular enzyme of the native host (Melasniemi, 1988). The sequence coding for the $\mathrm{N}$ terminal end of this protein started some 500 nucleotides after the apu open reading frame shown in Fig. 1. Thus, the enzyme and the satellite protein are coded by two adjacent, apparently co-regulated genes.

Purified extracellular $\alpha$-amylase-pullulanase of $C$. thermohydrosulfuricum (Melasniemi, 1988) contained $11-12 \%$ carbohydrate stainable after SDS-PAGE, suggesting a covalent linkage between the polypeptide and the carbohydrate moiety. In $N$-glycosidic linkages of glycoproteins the amino acid sequence next to the glycosylated asparagine is always Asn-X-Ser/Thr (Sharon \& Lis, 1981). The deduced amino acid sequence of the apu product revealed in total 13 such possible sites for $\mathrm{N}$-glycosylation (Fig. 1).

The hydropathy plot (Kyte \& Doolittle, 1982) of the apu product showed that the signal sequence is the most hydrophobic portion of the protein. No other regions having the length and the hydrophobicity to span a membrane (Kyte \& Doolittle, 1982) were found. Thus, it seems that the localization of the enzyme to the surface of $C$. thermohydrosulfuricum cells under some cultural conditions (Melasniemi, 1987), is by other means than via a membrane anchor sequence in the mature polypeptide.

\section{Codon usage}

The $37 \mathrm{~mol} \% \mathrm{G}+\mathrm{C}$ content of the apu gene agrees well with the value of $36-38 \mathrm{~mol} \%$ reported for several strains 
of C. thermohydrosulfuricum (Wiegel et al., 1979). However, the $\mathbf{G}+\mathbf{C}$ content of the third ('wobble') codon position was only $27 \%$, indicating a preference for codons ending in $\mathrm{A}$ or $\mathrm{T}$.

The codon usage of the apu gene resembled that observed for Clostridium thermocellum genes (Schwarz et al., 1988; Grépinet et al., 1988). It was biased and different from the codon usage in the structural genes of $E$. coli (Alff-Steinberger, 1984): for only three out of 17 amino acids (excluding Met, Trp and Cys, the last of which is absent from the protein) was the preferred codon the same as in the $E$. coli genes (data not shown). The preferred $a p u$ codons were more often those found in rare proteins of $E$. coli than those found in abundant proteins (Grosjean \& Fiers, 1982) and in addition the codons preferred in apu for Arg (AGA and AGG), Ile (ATA) and Gly (GGA) have been proposed as slowing down modulators of translation in $E$. coli (Grosjean \& Fiers, 1982). The exceptional length of the apu gene together with the differences in the codon usage presumably makes translation of the gene in $E$. coli slow and prone to premature termination, which, in turn, renders the newly synthesized polypeptides susceptible to proteolytic degradation. These factors may explain the observed degeneracy of the apu product in $E$. coli (Melasniemi \& Paloheimo, 1989). In contrast to the differences in codon usage between $a p u$ and $E$. coligenes, a resemblance was observed to the codon usage of Bacillus genes (McConnell et al., 1986): 14 amino acids out of the 17 shared a common preference codon in apu and in Bacillus genes.

\section{Similarities between the apu product and other amylolytic enzymes}

No general sequence similarity has been found in prokaryotic or eukaryotic signal sequences (McConnell et al., 1986). However, the signal sequence of the apu product showed similarity to the signal sequence of the $\alpha$ amylase of $B$. stearothermophilus, another Gram-positive thermophile, and to that of B. subtilis (Fig. 2a).

The deduced amino acid sequence of the mature $a p u$ product showed several regions similar to sequences in other amylolytic enzymes (Fig. 2; references in the legend). Nakajima et al. (1986) have demonstrated four common regions in $\alpha$-amylases of different origins. The first, second and fourth regions can also be detected in other types of amylolytic enzymes (see e.g. Kuriki \& Imanaka, 1989), and these could likewise be distinguished in the mature apu product (Fig. $2 e, g, j$ ). Most interestingly, considering the dual $\alpha-1,4 / \alpha-1,6$ activity of the apu product, the second and fourth regions were partly 'duplicated'. Thus, within these regions were sequence motifs common to several $\alpha$-amylases (shaded in Fig. $2 g$ and $j$ ) which were not found in the apu product at this position, but were found later in the $a p u$ sequence (Fig. $2 i, l$ ). The 78 amino acid sequence ending immediately after the first region (Fig. 2e) had 63\% identity with the amino acid sequence of the neopullulanase of $B$. stearothermophilus. The beginning of this sequence was also similar to the sequences of the isoamylase (EC 3.2.1.68) of Pseudomonas amyloderamosa, the pullulanase of 'Klebsiella aerogenes' and the cyclomaltodextrin glucanotransferases (EC 2.4.1.19) of $K$. pneumoniae and $B$. macerans. Somewhat later the sequence was similar to the $\alpha$-amylase of $B$. subtilis. The 57 amino acid sequence starting one amino acid before the second region had $53 \%$ identity with $B$. stearothermophilus neopullulanase (Fig. 2 g). Only one amino acid at and two amino acids near the third region (most obvious in mammalian $\alpha$-amylases) were present in the apu product (Fig. $2 h$ ). The sequence starting at the fourth region again resembled that of $B$. stearothermophilus neopullulanase (Fig. $2 j$ ) and was followed at some distance by a sequence with much similarity also to the cyclomaltodextrin glucanotransferases of $K$. pneumoniae and $B$. macerans (Fig. $2 k$ ).

As a whole, the apu product most closely resembled the neopullulanase of $B$. stearothermophilus, an enzyme cleaving $\alpha-1,4$ linkages in pullulan and also $\alpha-1,6$ linkages in several oligosaccharides (Imanaka \& Kuriki, 1989). Several short sequences were also very similar to sequences in $B$. macerans cyclomaltodextrin glucanotransferase, of which enzyme two end products $(\beta$ - and $\gamma$ cyclodextrins) are inhibitors of the $\alpha$-amylase-pullulanase (H. Melasniemi, unpublished). There were no sequences common to all three debranching enzymes, $P$. amyloderamosa isoamylase, ' $K$. aerogenes' pullulanase and the apu product, that distinguished them from other amylases, except that they all had a short hydrophobic stretch of amino acids near their ends (Fig. $2 n$ ) and terminated shortly after this with a basic residue.

The sequence of the C-terminal end of the apu product (Fig. 1) is of interest. The prominent sequence of three hydroxyl-bearing Thr residues alternating with hydrophobic amino acids (starting 35 residues before the end) followed shortly after by four Leu residues and four GIn residues, the whole sequence being punctuated and surrounded with several Lys Arg and His residues, is likely to serve a specific function. Because of its hydrophilicity this region is likely to be located on the surface of the molecule, where its positive charge and the hydroxyl groups might participate in the attachment of the enzyme to a negatively charged domain on the surface of $C$. thermohydrosulfuricum cells, on another $\alpha$ amylase-pullulanase polypeptide (dimerization or aggregation) or on the satellite protein. However, the presence of several His residues (otherwise the rarest of the amino 
GTG TTT AAA AGG AGA GCT TTA GGA TTT TTG CTG GCT TTT CTP TTA GTT TTT ACA GCA GTA TTT GGC TCA ATG CCT ATG GAA TTT GCA AAG

-31 Met Phe Lys Arg Arg Ala Leu Gly Phe Leu Leu Ala Phe Leu Leu Val Phe Thr Ala Val phe Gly Ser Met Pro Met Glu Phe Ala Lys GCT GAG ACA GAT ACA GCG CCA GCG ATA GCC AAT GTT GTT GGC AAT TTT CAA TCC AAA CTT GGA GAT TCT GAC TGG AAT ATA AAC AGT GAC

-1 Ala Glu Thr Asp Thr Ala Pro Ala Ile Ala Asn Val Val Gly Asn Phe Gln Ser Lys Leu Gly Asp Ser Asp Trp Asn Ile Asn Ser Asp AAA ACG ATA ATG ACA TAT AAA GGT AAT GGC TTT TAT GAA TTT ACT ACC CCA GTT GCG TTA CCT GCA GGT GAT TAT GAG TAT AAA GTT GCi

30 Lys Thr Ile Met Thr Tyr Lys Gly Asn Gly Phe Tyr Glu Phe Thr Thr Pro Val Ala Leu Pro Ala Gly Asp Tyr Glu Tyr Lys Val Ala LindIII

CTT AAT CAT TCA TGG GAA GGT GGA GGA GTT CCT TCA CAA GGT AAT TTA AGC TTT CAT CTT GAT TCA GAT TCT GTA GTG ACT TTT TAT TAC

60 Leu Asn His Ser Trp Glu gly Gly Gly Val Pro Ser Gln Gly Asn Leu Ser phe His Leu Asp Ser Asp Ser Val Val Thr phe Tyr Tyr

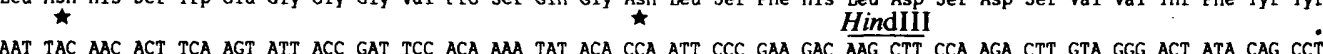

90 Asn Tyr Asn Thr Ser Ser Ile Thr Asp Ser Thr Lys Tyr Thr Pro Ile Pro Glu Asp Lys Leu Pro Arg Leu Val Gly Thr Ile Gln Pro GCA ATA GGA GCA GGT GAT GAT TGG AAA CCT GAA ACA TCG ACT GCT ATA ATG AGA GAC TAT AAG TTT AAT AAT GTT TAC GAA TAC ACT GCA 120 Ala Ile Gly Ala Gly Asp Asp Trp Lys Pro Glu Thr Ser Thr Ala Ile Met Arg Asp tyr Lys phe Asn Asn Val Tyr Glu Tyr Thr Ala AAT GTT CCA AAA GGG AAT TAT GAG TTT AAA GTG ACT TTA GGG CCC TCA TGG GAT ATA AAT TAT GGC TTA AAT GGT GAA CAA AAT GGG CCA 150 Asn Val Pro lys Gly asn Tyr Glu phe lys Val Thr Leu Gly Pro ser trp Asp Ile Asn tyr Gly Leu Asn Gly Glu Gln Asn Gly Pro AAT ATT CCT TTG AAT GTA GCC TAT GAT ACT AAG ATT ACA TTT TAC TAT GAT TCG GTT TCA EAT AAT aTA TGG aCA GAT TAT aAT CCA CCI 180 Asn Ile Pro Leu Asn Val Ala Tyr Asp Thr Lys Ile Thr Phe Tyr Tyr Asp Ser Val Ser His Asn Ile Trp Thr Asp Tyr Asn Pro Pro CTT ACA GGA CCT GAT AAT AAC ATA TAT TAT GAC GAT TTA AGA CAT GAC ACC CAT GAC CCA TTC TTC CGC TCG CCT TTC GGT GCA ATA AAA 210 Leu Thr Gly Pro Asp Asn Asn Ile Tyr Tyr Asp Asp Leu Arg His Asp Thr His Asp pro Phe Phe Arg Ser Pro Phe Gly Ala Ile Lys ACG GGA GAT ACT GTG ACA TTA AGA ATA CAA GCA AAA AAT CAT GAC ATT GAG TCA GCT AAA ATT TCT TAT TGG GAT GAT ATT AAA AAA ACA 240 Thr Gly Asp Thr Val Thr Leu Arg Ile Gin Ala Lys Asn His Asp Ile Glu Ser Ala Lys Ile Ser Tyr Trp Asp Asp Ile Lys Lys Thr HindIII

AGA ATA GAA GTC CCT ATG TAC AGA ATT GGT CAA AGT CCT GAC GGG AAA TAT GAA TAC TGG GAA GTT Aag TTA AGC TTT GAC CAT CCC ACA 270 Arg Ile Glu val Pro Met Tyr Arg Ile Gly Gln Ser Pro Asp Gly Lys Tyr Glu Tyr Trp Glu Val Lys Leu Ser Phe Asp His Pro Thr AGA ATT TGG TAT TAC TTT ATA CTT AAA GAC GGG ACC AAA ACT GCT TAT TAC GGA GAT AAC GAT GAA CAA TTA GGT GGC GTA GGT AAA GCC 300 Arg Ile Trp Tyr Tyr phe Ile Leu Lys Asp Gly Thr Lys Thr Ala Tyr Tyr Gly Asp Asn Asp Glu Gln Leu Gly Gly Val Gly Lys Ala Arg Tle TrP Tyr Tyr Phe Tle Leu Lys Asp Gly Thr Lys

ACA GAT ACA GAA AAT AAG GAC TTC GAA CTT ACT GTC TAC GAT AAA AAC TTA GAC ACC CCT GAT TGG ATG AAA GGG TCA GTA ATG TAT CAA

330 Thr Asp Thr Glu Asn Lys Asp phe Glu Leu Thr Val Tyr Asp Lys Asn Leu Asp Thr Pro Asp Trp Met Lys Gly Ser Val Met Tyr Gin

ATA TTC CCT GAT AGG TTC TTT AAT GGG GAT TCT TCA AAT GAC CAT CTA AAG AAA TAC AGC AGA GGT TTT GAT CCT GTT GAA TAT CAT AGC 360 Ile phe Pro Asp Arg phe Phe Asn Gly Asp Ser Ser Asn Asp His Leu Lys Lys Tyr Ser Arg Gly Phe Asp Pro Val Glu Tyr His Ser

AAC TGG TAC GAG CTT CCA GAT AAT CCG AAT GAT AAA AAT AAA CTA GGA TAT ACA GGG GAT GGC ATA TGG TCA AAT GAC TTC TTT GGC GGT

390 Asn trp tyr glu Leu Pro Asp Asn Pro Asn Asp Lys Asn lys leu gly Tyr thr gly asp gly Ile Trp Ser Asn Asp Phe phe Gly Gly

GAT TTA AAA GGT ATA GAT GAT AAA TTG GAT TAT TTA AAA AGC CTT GGA ATA TCA GTC ATT TAT CTC AAT CCA ATT TTT CAA TCA CCT TCT

420 Asp Leu Lys Gly Ile Asp Asp Lys Leu Asp Tyr Leu lys Ser Leu Gly Ile Ser Val Ile tyr Leu Asn Pro Ile Phe Gln Ser Pro Ser

AaT CAC AGG TAC GAT ACA ACC GAT TAC ACA AAA ATA GAC GAA CTA TTA GGA GAT TTA TCT ACC TTT Aag Aag CTC aTG GAG GAT GCC CAT

450 Asn His Arg Tyr Asp Thr Thr Asp Tyr Thr Lys Ile Asp Glu Leu Leu Gly Asp Leu Ser Thr Phe Lys Lys Leu Met Glu Asp Ala His

GCA AAA GGG ATT AAG GTA ATA CTT GAT GGC GTC TTC AAT CAT ACA AGT GAT GAC AGC ATT TAT TTT GAT AGA TAT GGT AAA TAC TTA AAC

480 Ala Lys Gly Ile Lys Val Ile Leu Asp Gly Val Phe Asn His Thr Ser Asp Asp Ser Ile Tyr Phe Asp Arg Tyr Gly Lys Tyr Leu Asn ACG GGA GTT TTA GGT GCT TAT CAA GCA TGG AAA CAG GGA GAT CAG TCA AAG TCT CCA TAT GGT GAC TGG TAT GAG ATA AAG CCT GAC GGT

510 Thr Gly Val Leu Gly Ala Tyr Gln Ala Trp Lys Gln Gly Asp GIn Ser Lys Ser Pro Tyr Gly Asp Trp Tyr Glu Ile Lys Pro Asp Gly

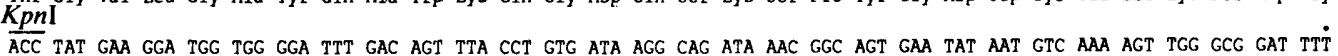

540 Thr Tyr Glu Gly Trp Trp Gly phe Asp Ser Leu Pro Val Ile Arg Gln Ile Asn Gly Ser Glu Tyr Asn Val Lys Ser Trp ala Asp phe

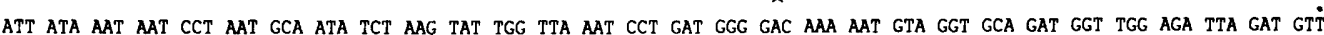

570 Ile Ile Asn Asn Pro Asn Ala Ile Ser Lys Tyr Trp Leu Asn Pro Asp Gly Asp Lys Asn Val Gly Ala Asp Gly Trp Arg Leu Asp Val GCG AAT GAA GTT GCT CAC GAT TTT TGG GTT CAT TTT AGA GGT GCA ATT AAT ACT GTG AAA CCG AAT GCT CCG ATG GTT GCA GAA AAC TGG 600 Ala Asn Glu Val Ala His Asp phe Trp Val His Phe Arg Gly Ala Ile Asn Thr Val Lys Pro Asn Ala Pro Met Val Ala Glu Asn Trp AAT GAC GCT TCA CTG GAT CTG CTT GGA GAT TCT TTT AAC TCT GTT ATG AAT TAT CTC TTT AGA AAT GCA GTA ATT GAC TTT ATA TTG GAT 630 Asn Asp Ala Ser Leu Asp Leu Leu Gly Asp Ser phe Asn Ser Val Met Asn Tyr Leu Phe Arg Asn Ala Val Ile Asp phe Ile Leu Asp

AAA TCA TTT GAT GAC GGA AAT GTG GTT CAC AAT CCT ATA GAT GCA GCA AAA CTC GAC CAA AGG CTT ATG AGC ATA TAT GAG AGA TAT CCT 660 Lys Ser Phe Asp Asp Gly Asn Val Val His Asn Pro Ile Asp Ala Ala Lys Leu Asp Gln Arg Leu Met Ser Ile Tyr Glu Arg Tyr Pro 


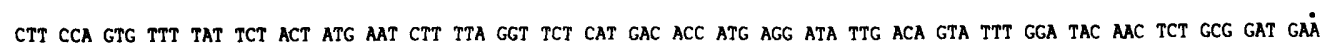
690 Leu Pro Val Phe Tyr Ser Thr Met Asn Leu Leu Gly Ser His Asp Thr Met arg Ile Leu Thr Val Phe Gly Tyr Asn Ser Ala Asp GIu AAT CAA AAT TCT CAA GCA GCA AAA GAC CTT GCG GTT AAA AGA CTT AAA CTT GCT GCA ATA CTG CAA ATG GGT TAT CCA GGC ATG CCT TCT 720 Asn Gln Asn Ser Gln. Ala Ala Lys Asp Leu Ala Val Lys Arg Leu Lys Leu Ala Ala Ile Leu Gln Met Gly Tyr Pro Gly Met Pro Ser

ATT TAT TAC GGA GAT GAA GCA GGA CAA TCT GGC GGA AAA GAC CCG GAC AAC AGA AGG ACA TTC CCT TGG GGA AGA GAA GaT ACT GAT TTG 750 Ile Tyr Pyr Gly Asp Glu Ala Gly Gln Ser Gly Gly Lys Asp Pro Asp Asn Arg Arg Thr Phe Pro Trp Gly Arg Glu Asp Thr Asp Leu CAA ACT TTT TTC AAG AAA GTT GTA AAT ATA AGA AAT GAG AAT CAA GTT TTG AAA ACA GGA GAC CTT GAG ACA CTT TAT GCA AAT GGC GAं 780 Gln Thr phe phe Lys Lys Val Val Asn Ile Arg Asn Glu Asn Gln Val Leu Lys Thr Gly Asp Leu glu Thr Leu Tyr Ala Asn Gly Asp

GTT TAT GCC TTT GGA AGA AGA ATC ATA AAT GGA AAA GAT ACT TTT GGG AAA TCG TAT CCT GAC AGT GTA GCT ATT GTC GTA ATA AAT AAA 810 Val Tyr Ala phe Gly Arg Arg Ile Ile Asn Gly Lys Asp Thr phe Gly Lys Ser Tyr Pro Asp Ser Val Ala Ile Val Val Ile Asn Lys GGT GAC GCA AaA CAG GTT TCT ATA GAT ACC ACT AAA TTT ATA AGA GAT GGA GTT GCT TTT ACA GAT GCC TTA aGT GGC AaG aCA TAC ACG 840 Gly Asp Ala Lys Gin Val Ser Ile Asp Thr Thr Lys phe Ile Arg Asp Gly Val Ala phe Thr Asp Ala Leu Ser Gly Lys Thr Tyr Thr GTT CAG GAT GGT AAA ATT GTT GTA GAA GTT GGA TCA ATG GAT GGA GCT ATA CTT ATA TCA GAT ACA GGA CAA AAT TTG ACA GCA CCT CÁ 870 Val Gln Asp Gly Lys Ile Val Val Glu Val Gly Ser Met Asp Gly Ala Ile beu Ile Ser Asp Thr Gly Gln Asn Leu Thr Ala Pro Gln CCA ATA ACA GAC CTT AAA GCA GTC TCA GGG AAT GGC AAA GTA GAC CTT TCC TGG AGT GTA GTA GAT AAA GCA GTA AGC TAT AAT ATT TAC 900 Pro Ile Thr Asp Leu Lys Ala Val Ser Gly Asn Gly Lys Val Asp Leu Ser Trp Ser Val Val Asp Lys Ala Val Ser Tyr Asn Ile Tyr CGT TCT ACA GTT AAA GGT GGG TTA TAT GAA AAA ATA GCT TCA AAT GTT ACG CAA ATT ACC TAT ACT GAT ACA GAG GTT ACC AAT GGT CTA 930 Arg Ser Thr Val Lys Gly Gly Leu Tyr Glu Lys Ile Ala Ser Asn Val thr Gin tle Thr Tyr Thr Asp Thr Glu Val Thr Asn Gly Leu Aag TAT GTG TAT GCT GTA ACC GCT GTA GAT AAT GAT GGA AAT GAG AGT GCT TTA AGC AAT GAA GTT GAG GCA TAT CCA GCA tTT CCT aTT 960 Lys Tyr Val Tyr Ala Val Thr Ala Val Asp Asn Asp Gly Asn Glu Ser Ala Leu Ser Asn Glu Val Glu Ala Tyr Pro Ala Phe Pro Ile GGC TGG GCG GGA AAT ATG AAT CAA GTA AAT ACA CAT GTA ATA GGT GTA AAT AAT CCA GTT GAA GTG TAT GCT GAA GTG TGG GCT CAA GGG 990 Gly Trp Ala Gly Asn Met Asn Gln Val Asn Thr His Val Ile Gly Val Asn Asn pro Val Glu Val Tyr Ala Glu Val Trp ala Gln Gly CTT ACA GAT AAA CCT GGC CAA GGA GAA AAT ATG ATT GCC CAG TTA GGA TAT AGG TAT ATT GGA GAT ACT GTA GGA GAT GCA GTT TAC AAT 1020 Leu Thr Asp Lys Pro Gly Gln Gly Glu Asn Met Ile Ala Gln Leu Gly Tyr Arg Tyr Ile Gly Asp Thr Val Gly Asp Ala Val Tyr Asn GCC GTG TAC AAT AAG GTA GAA GGT GTT GAA ATA AGT AAG GAC TGG ACA TGG GTT GAT GCA CAA TAT GTA GGT GAT TCT GGA AAT AAT GAT 1050 Ala Val Tyr Asn Lys Val Glu Gly Val Glu Ile Ser Lys Asp Trp Thr Trp Val Asp Ala Gln Tyr Val Gly Asp Ser Gly Asn Asn Asp

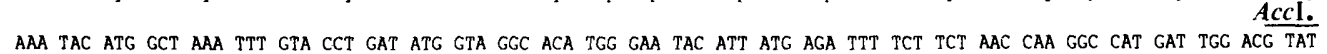
1080 Lys Tyr Met Ala lys phe Val Pro Asp Met Val Gly Thr Trp Glu Tyr Ile Met arg phe Ser Ser Asn Gln Gly His Asp Trp Thr Tyr ACA AAA GGA CCA GAT GGG AAA ACA GAT GAA GCA AAA CAG TTT ACT GTC GTG CCA TCA AAT GAT GTA GAA ACA CCT ACA GCT CCA GTC TTA 1110 Thr Lys Gly Pro Asp Gly lys Thr Asp Glu Ala Lys Gln Phe Thr Val Val Pro Ser Asn Asp Val Glu Thr Pro Thr Ala Pro Val Leu CAA CAA CCA GGA ATT GAA TCC TCA AGA GTT ACA CTT AAC TGG AGT CCG TCA GCT GAT GAT GTT GCT ATT TTC GGC TAC GAA ATC TAT AAG 1140 Gln Gln Pro Gly Ile Glu Ser Ser Arg Val Thr Leu Asn Trp Ser Pro Ser Ala Asp Asp Val Ala Ile phe Gly Tyr Glu Ile Tyr Lys TCT TCA AGT GAG ACA GGA CCA TTT ATA AAG ATT GCA ACT GTG TCT GAC AGT GTG TAT AAC TAC GTA GAT ACA GAT GTA GTA AAT GGC AAT 1170 Ser Ser Ser Glu Thr Gly Pro phe Ile Lys Ile Ala Thr Val Ser Asp Ser Val Tyr Asn tyr Val Asp Thr Asp Val Val Asn Gly Asn

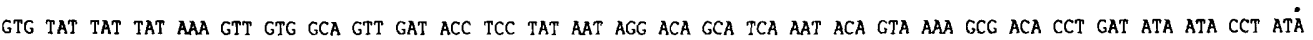
1200 Val Tyr Tyr Tyr Lys Val Val Ala Val Asp Thr Ser Tyr Asn Arg thr Ala Ser Asn thr Val Lys Ala Thr pro Asp Ile Ile Pro Ile AAA GTG ACA TTT AAT GTC ACA ATA CCA GAT TAT ACT CCT GAT GAT GGT GTA AAT ATT GCT GGA AAC TTC CCG GAT GCT TTC TGG AAT CCA 1230 Lys Val Thr Phe Asn Val Thr Ile Pro Asp Tyr Thr Pro Asp Asp Gly Val Asn Ile Ala Gly Asn Phe Pro Asp Ala Phe Trp Asn Pro Aat GCT AAT CAA ATG ACA AAA GCT GGT TCT AAC ACC TAT AGT ATT ACA CTT ACT TTA AAT GAG GGT ACA CAA aTT GAA TAT aAA TAT GCA 1260 Asn Ala Asn Gin Met Thr Lys Ala Gly Ser Asn Thr Tyr Ser Ile Thr Leu Thr Leu Asn Glu Gly Thr Gin Ile Glu Tyr Lys Tyr Ala AGG GGG AGC TGG GAT AAA GTA GAA AAA GGT GAA TAC GGC AAT GAG ATT GAC AAC AGA AAA ATA ACT GTT GTC AAT CAG GGT TCA AAT ACA 1290 Arg Gly Ser Trp Asp Lys Val Glu lys Gly Glu tyr Gly Asn Glu Ile Asp Asn Arg Lys Ile thr Val Val Asn Gln Gly Ser Asn Thr ATG GTT GTA AAT GAT ACA GTG CAA AGA TGG AGA GAT GTG CCA ATA TAT ATC TAT TCT CCA AAA GAC aAG ACT ATT GTT GAT GCA AAT ACG 1320 Met Val Val Asn Asp Thr Val Gln Arg Trp Arg Asp Val Pro Ile Tyr Ile Tyr Ser Pro Lys Asp Lys thr Ile Val Asp ala Asn Thr AGT GAA ATA GAG ATT AAA GGC aAT aCT TAC AAA GGT GCG AAA GTA ACT ATA AAT GAT GAA TCT TTT GTG CAA CAA GAA AAT GGA GTA TT' 1350 Ser Glu Ile Glu Ile lys Gly Asn Thr Tyr bys Gly Ala Lys Val thr Ile Asn Asp glu Ser phe val Gln Gln Glu Asn Gly Val phe ACA AAA GTA GTT CCC TTG GAA TAT GGT GTA AAT ACT ATC AAA ATA CAT GTA GAG CCA AGT GGT GAC AaG AAT AAT GAA CTT ACA AAA GAT 1380 Thr Lys Val Val Pro leu Glu tyr Gly Val Asn Thr lle lys lle his Val Glu Pro Ser Gly Asp bys Asn Asn Giu Leu Thr Lys Asp ATA ACA ATA ACT GTT ACA AGA GAG AAG CCG GCC AGG AGA CAG AaC CTA CTC CTA CTC CAC CAA CAG aAa CAA CAA AAC CAT CTC AAG AAG 1410 Ile Thr Ile thr Val thr arg Glu Lys Pro Ala Arg Arg Gin Asn Leu leu leu Leu His Gin Gin Lys Gin Gin Asn His Leu Lys Lys TAT CAC AAG GCA AAA TAGTTGTAGAAAATAACACAACAACACTTACAATAGACGAAAACAAAGTAGCAAAAGAACATAAAAGACACTTCAAAGAAAGAAATACGATTTGAC 1440 Tyr His Lys Ala Lys End End

$$
\text { End End }
$$

2568

Fig. 1. Nucleotide and deduced amino acid sequence of the apu gene. Putative promoter and ribosome-binding sites are denoted by double and single underlinings, respectively. Inverted repeats are denoted by arrows and the $\mathbf{N}$-terminal end of the mature protein is underlined. Restriction sites present also in the polylinker of the M13 vector are shown above the sequence. Possible $N$-glycosylation sites are shown by an asterisk. 
(a) Cth apu

Bst amy

Bsu amy

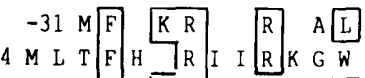

-33 M E A K R F K T S L D

G F L L A F L L V F T A V F G M P ME F A L A $M$ F L L L A F

(c)

Cth'apu 240 T G T V T L 247 Shy amy $392 \mathrm{D} G \mathrm{D}$ T V T V G 399 Bma cgt 584 T G D Q T V R 591

\section{(d)}

Cth apu 356 V M Y Q I F P D R F F N G DS S N 372 Bst npl 387 V W W Y BarAamy 319 G I P C I F Y D 固 F F N W G F K D 335 Bma cgt 16 V I Y Y I V T D R F A D G D R T N 32 (e)

Cth apu Bst npl Pam iam Kae pul Bsu amy Shy amy Kpn cgt Bma cgt

Cth apu Bst npl Pam iam Kae pul Bsu amy Shy amy

(g) Cth apu Bst npl Pam iam Kae pul Bsu amy Bam amy Shy amy sli amy

\begin{tabular}{|c|c|}
\hline (h) & 3 \\
\hline apu & $634 \mathrm{~L}$ D I L 638 \\
\hline amy & \begin{tabular}{ll|ll|l|l|l}
232 & $\mathrm{I}$ & $\mathrm{D}$ & $\mathrm{L}$ & $\mathrm{G}$ & $\mathrm{G}$ & 236
\end{tabular} \\
\hline amy & 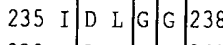 \\
\hline & $238 \mathrm{I} D \mathrm{D} L \mathrm{~L} G 241$ \\
\hline
\end{tabular}

(i) \begin{tabular}{ll|llll} 
Cth apu & 672 & I D A A K & 676 \\
Bam amy & 230 & I & D A A K & 234 \\
Shy amy & 173 & I & D A A K & 177 \\
Sli amy & 176 & I D D A A K & 180
\end{tabular} (f)

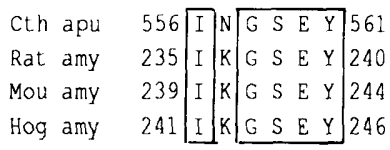

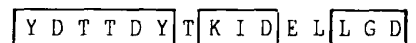

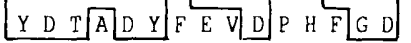
170 E F G G D L Q G I I D H L D Y L V D L G I T G I Y L T T P I F R S P S N H]K 198 Q Y R G TY Y G A G L K A S Y L A S L G V 218

$40 \mathrm{SP}$ I N Q $\mathrm{VKK}$ E G $\mathrm{N}$ C G D K SM SNW Y W L Y P P TSYQ I G N R Y L G T

$47 Y \mathrm{~T}$ GGDLRGLIN KLP YLKSLG V 67

60 K I A G R L G D 49 Y E G G D W Q G I I D D K I N D G Y L T G M 69
L S TFKK L M E D AHAKGIKVILDGV VF H T SD 496

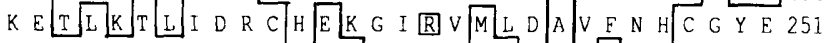
281 F H N A A G I K V Y Y M D V V V Y Y N H T T A E G 300 590 K Q D L G MN $\cup$ I M D D V V Y Y N H T A E G 609 R D A E $\sqrt{A} S$ M V S A C HA A G V K V I A D A V V L H M A A G 97 E Q E E F K E M C A A A E E Y G I K V I V D A V V I N H T T T S D 106

\section{2}

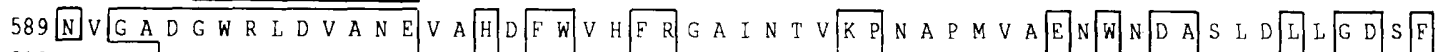
319 E F D I D G W R L D VAA N E I D H E F W R E ER Q E V K A L K P D V Y I L G E I W H D A M P W L R G D Q E 365 T M G D D F R FD DA S 378 666 D Y K I D D G F 167 ND GAD G F R F D A A K H 180 222 E L S L D D G F 165 S L G V D D G F R I I D A 168 S L G V D G F E I D D A A K H 18 ?

Cth apu N S V M N Y F 649

Bst npl D A V M N Y P 379 (j)

Cth apu Bst npl Pam iam Kae pul Bsu amy Bli amy Bst amy BarBamy Bma cgt

(k) Cth apu Bst npl Kpn cgt Bma cgt

(l) Cth apu Bli amy Bst amy BarBamy
746 GMSP IYYGDEA Ge S GGK D P D NR R T F EWGR E D 776

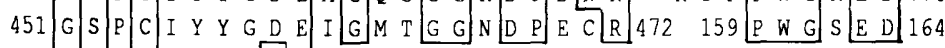

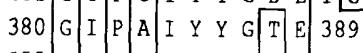

$355 \mathrm{G} V \mathrm{~V}$ A I I Y Y G TE

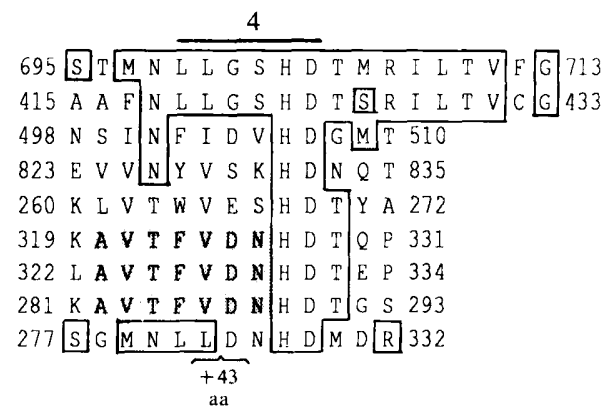
415 A A F N L L G S H D T S R I L T V C C 433 498 N S I N F I D V H D G M T 510 823 E V VLN Y V S K H D N Q T 835 260 K L V T W V E S H D T Y A 272 319 K A V T F V D N H D T Q P 331 322 L A V T F V D N H D T E P 334 281 K A V T F V D N H D T G S 293 $277 \mathrm{~S} G \underbrace{M N ~ L ~ L ~ D ~ N ~ D ~ M ~ D ~ R ~}_{+43} 332$

799 G D L E T L 804 220 G

(m)

Cth apu Rat amy Hum amy
$1 3 9 9 \longdiv { S G D K N } 1 4 0 4$ 452 S G D K V V N 457 459 S G D K I 464 (n)

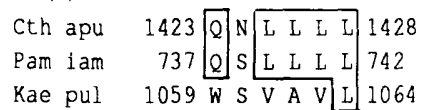


acids found in the $a p u$ product) raises the possibility that the end of the molecule might instead of serving a structural function be involved in substrate binding or even be part of the active site of the enzyme. Regions one, two and four common to $\alpha$-amylases (Nakajima $e t$ $a l ., 1986)$ all contain a His residue and it is assumed that in the $\alpha$-amylase of Aspergillus oryzae (Matsuura et al., 1984) two of these histidines are involved in substrate binding and one is at the active site. The $\mathrm{C}$-terminal end of the pullulanase of ' $K$. aerogenes' bears some resemblance to the end of the apu product. It is likewise enriched with basic amino acids, including two His residues. In addition, the four amino acid hydrophobic stretch near the end of the ' $K$. aerogenes' pullulanase is preceded by a Ser-Val-Thr-Leu sequence resembling the alternating sequence of hydroxy- and hydrophobic amino acids found near the end of the $a p u$ product. It will be interesting to see whether the sequences of other $\alpha$ amylase-pullulanase enzymes show 'duplication' of the second and fourth common regions and whether the Cterminal sequences of other ( $\alpha$-amylase-) pullulanases have characteristics in common with the $C$. thermohydrosulfuricum enzyme.

We thank Vesa Joutsjoki, MSc, for the synthetic oligonucleotides and Dr John Londesborough for critically reading the manuscript.

\section{References}

AlfF-STEINBerger, C. (1984). Evidence for a coding pattern on the non-coding strand of the E. coli genome. Nucleic Acids Research 12, 2235-2241.

amemura, A., Chakraborty, R., Fujita, M., Noumi, T. \& Futal, M. (1988). Cloning and nucleotide sequence of the isoamylase gene from Pseudomonas amyloderamosa SB-15*. Journal of Biological Chemistry 263, 9271-9275.

BiNDER, F., HUBER, O. \& BöCK, A. (1986). Cyclodextrin-glycosyltransferase from Klebsiella pneumoniae M5al: cloning, nucleotide sequence and expression. Gene 47, 269-277.

BIRNBOIM, H. C. \& Doly, J. (1979). A rapid alkaline extraction procedure for screening recombinant plasmid DNA. Nucleic Acids Research 7, 1513-1523.

Coleman, R. D., YANG, S.-S. \& MCAlister, M. P. (1987). Cloning of the debranching-enzyme gene from Thermoanaerobium brockii into Escherichia coli and Bacillus subtilis. Journal of Bacteriology 169, 4302-4307.
Crombrugghe, B., Busby, S. \& Buc, H. (1984). Cyclic AMP receptor protein: role in transcription activation. Science 224, 831-838.

FOGARTY, W. M. (1983). Microbial amylases. In Microbial Enzymes and Biotechnology, pp. 1-92. Edited by W. M. Fogarty. Barking: Applied Science Publishers.

Grépinet, O., Chebrou, M.-C. \& Béguin, P. (1988). Nucleotide sequence and deletion analysis of the xylanase gene (xynZ) of Clostridium thermocellum. Journal of Bacteriology 170, 4582-4588.

Grosjean, H. \& Firrs, W. (1982). Preferential codon usage in procaryotic genes: the optimal codon-anticodon interaction energy and the selective codon usage in efficiently expressed genes. Genes. 18, 199-209.

Hoshiko, S., Makabe, O., Nojiri, C., Katsumata, K., Satoh, E. \& NAGAOKA, K. (1987). Molecular cloning and characterization of the Streptomyces hygroscopicus $\alpha$-amylase gene. Journal of Bacteriology 169, 1029-1036.

HyUN, H. H. \& ZeIKus, J. G. (1985). Regulation and genetic enhancement of glucoamylase and pullulanase production in Clostridium thermohydrosulfuricum. Journal of Bacteriology 164, 11461152.

IMANAKA, T. \& KURIKI, T. (1989). Pattern of action of Bacillus stearothermophilus neopullulanase on pullulan. Journal of Bacteriology 171, 369-374.

Katsuragi, N., Takizawa, N. \& Murooka, Y. (1987). Entire nucleotide sequence of the pullulanase gene of Klebsiella aerogenes W70. Journal of Bacteriology 169, 2301-2306.

KURIKI, T. \& IMANARA, T. (1989). Nucleotide sequence of the neopullulanase gene from Bacillus stearothermophilus. Journal of General Microbiology 135, 1521-1528.

KYTE, J. \& DOoLITTLE, R. F. (1982). A simple method for displaying the hydropathic character of a protein. Journal of Molecular Biology 157, 105-132.

long, C. M., Virolle, M.-J., Chang, S.-Y., Chang, S. \& Bibb, M. J. (1987). $\alpha$-Amylase gene of Streptomyces limosus: nucleotide sequence, expression motifs, and amino acid sequence homology to mammalian and invertebrate $\alpha$-amylases. Journal of Bacteriology 169, 5745-5754.

Maniatis, T., Fritsch, E. F. \& Sambrook, J. (1982). Molecular Cloning: a Laboratory Manual. Cold Spring Harbor, NY: Cold Spring Harbor Laboratory.

MatsuUra, Y., Kusunoki, M., Harada, W. \& KaKudo, M. (1984). Structure and possible catalytic residues of Taka-amylase A. Journal of Biochemistry 95, 697-702.

McConnell, D. J., Cantwell, B. A., Devine, K. M., Forage, A. J., LAoide, B. M., O'Kane, C., Ollington, J. F. \& SharP, P. M. (1986). Genetic engineering of extracellular enzyme systems of bacilli. In Biochemical Engineering IV (Annals of the New York Academy of Sciences 469), pp. 1-17. Edited by H. C. Lim \& K. Vankatasubramanian. New York: New York Academy of Sciences.

MELASNIEMI, H. (1987). Effect of carbon source on production of thermostable $\alpha$-amylase, pullulanase and $\alpha$-glucosidase by Clostridium thermohydrosulfuricum. Journal of General Microbiology 133, 883-890.

Melasniemi, H. (1988). Purification and some properties of the extracellular $\alpha$-amylase-pullulanase produced by Clostridium thermohydrosulfuricum. Biochemical Journat 250, 813-818.

Fig. 2. Amino acid sequence similarities between the $a p u$ product and various amylolytic enzymes. (a) Signal sequence. $(b-n)$ Mature protein. Only those motifs found in addition to the apu product in at least two of the enzymes tested are shown. Abbreviations and references: apu, $\alpha$-amylase-pullulanase; amy, $\alpha$-amylase; npl, neopullulanase; iam, isoamylase; pul, pullulanase; cgt, cyclomaltodextrin glucanotransferase; Cth (Clostridium thermohydrosulfuricum) apu (this work); Bst (Bacillus stearothermophilus) amy (referred to by Nakajima et al., 1986, as all others denoted by an asterisk); Bsu (Bacillus subtilis) amy (*); Bam (Bacillus amyloliquefaciens) amy (*); Bli (Bacillus licheniformis) amy (Yuuki et al., 1985); Shy (Streptomyces hygroscopicus) amy (Hoshiko et al., 1987); Sli (Streptomyces limosus) amy (Long et al., 1987); Bar (barley) Aamy and Bamy (amylases A and B; Rogers, 1985); Rat amy (*); Mou (mouse pancreas) amy (*); Hog (pancreas) amy (*); Hum (human salivary) amy (*); Bst npl (Kuriki \& Imanaka, 1989); Kae ('Klebsiella aerogenes' [K. pneumoniae]) pul (Katsuragi et al., 1987); Pam (Pseudomonas amyloderamosa) iam (Amemura et al., 1988); Kpn (Klebsiella pneumoniae) cgt (Binder et al., 1986); and Bma (Bacillus macerans) cgt (Takano et al., 1986). Numbering starts from the first amino acid of the mature proteins. Numbered bars above the sequence denote common regions in $\alpha$-amylases (Nakajima et al., 1986) and shading denotes sequence motifs 'duplicated' in the apu product. 
Melasniemi, H. \& Paloheimo, M. (1989). Cloning and expression of the Clostridium thermohydrosulfuricum $\alpha$-amylase-pullulanase gene in Escherichia coli. Journal of General Microbiology 135, 1755-1762.

Messing, J. (1983). New M13 vectors for cloning. Methods in Enzymology 101, 20-78.

Nakajima, R., Imanaka, T. \& Aiba, S. (1986). Comparison of amino acid sequences of eleven different $\alpha$-amylases. Applied Microbiology and Biotechnology 23, 355-360.

Norrander, J., Kempe, T. \& Messing, J. (1983). Construction of improved M13 vectors using oligodeoxynucleotide-directed mutagenesis. Gene 26, 101-106.

Plant, A. R., Clemens, R. M., Morgan, H. W. \& Daniel, R. M. (1987). Active-site- and substrate-specificity of Thermoanaerobium Tok6-B1 pullulanase. Biochemical Journal 246, 537-541.

Rogers, J. C. (1985). Two barley $\alpha$-amylase gene families are regulated differently in aleurone cells. Journal of Biological Chemistry 260, 3731-3738.

ROSENBERG, M. \& COURT, D. (1979). Regulatory sequences involved in the promotion and termination of RNA transcription. Annual Review of Genetics 13, 319-353.

SANGer, F., NickLen, S. \& Coulson, A. R. (1977). DNA sequencing with chain-terminating inhibitors. Proceedings of the National Academy of Sciences of the United States of America 74, 5463-5467.

Sata, H., Umeda, M., Kim, C.-H., TANiguchi, H. \& Maruyama, Y. (1989). Amylase-pullulanase enzyme produced by $B$. circulans F-2. Biochimica et Biophysica Acta 991, 388-394.
Schwarz, W. H., Schimming, S., Rücknagel, K. P., BurgschWaiger, S., Kreil, G. \& Staudenbauer, W. L. (1988). Nucleotide sequence of the celC gene encoding endoglucanase $\mathrm{C}$ of Clostridium thermocellum. Gene 63, 23-30.

SHARON, N. \& LIS, H. (1981). Glycoproteins: research booming on long-ignored, ubiquitous compounds. Chemical \& Engineering News 30, $21-44$.

Takano, T., Fukuda, M., Monma, M., Kobayashi, S., Kainuma, K. \& YAMANE, K. (1986). Molecular cloning, DNA nucleotide sequencing, and expression in Bacillus subtilis cells of the Bacillus macerans cyclodextrin glucanotransferase gene. Journal of Bacteriology 166, $1118-1122$.

TAKasaki, Y. (1987). Pullulanase-amylase complex enzyme from Bacillus subtilis. Agricultural and Biological Chemistry 51, 9-16.

Wiegel, J., LJUNGDAHL, L. G. \& RAwSON, J. R. (1979). Isolation from soil and properties of the extreme thermophile Clostridium thermohydrosulfuricum. Journal of Bacteriology 139, 800-810.

YanisCh-Perron, C., Vieira, J. \& Messing, J. (1985). Improved M13 phage cloning vectors and host strains: nucleotide sequence of the M13mp18 and pUC19 vectors. Gene 33, 103-119.

Yuuki, T., Nomura, T., Tezuka, H., Tsubol, A., Yamagata, H., TsuKagoshi, N. \& UdAKa, S. (1985). Complete nucleotide sequence of a gene coding for heat- and pH-stable $\alpha$-amylase of Bacillus licheniformis: comparison of the amino acid sequences of three bacterial liquefying $\alpha$-amylases deduced from the DNA sequences. Journal of Biochemistry 98, 1147-1156. 